\title{
THE EXTENSION OF THE HUBBLE DIAGRAM. I. NEW REDSHIFTS AND $B V R$ PHOTOMETRY OF REMOTE CLUSTER GALAXIES, AND AN IMPROVED RICHNESS CORRECTION
}

\author{
Allan Sandage, Jerome Kristian, and James A. Westphal \\ Hale Observatories, Carnegie Institution of Washington and California Institute of Technology \\ Received 1975 September 5
}

\begin{abstract}
Absorption-line redshifts for 37 galaxies in 31 remote clusters have been measured with the new sky-subtracting prism spectrograph, using an SIT television detector with digital readout. Twentyfive galaxies in the sample have redshifts larger than $z=0.20$. New photoelectric $B V R$ photometry has been obtained for 16 of the clusters. Magnitudes corrected for aperture effect, $K$-dimming, galactic absorption, cluster richness, and Bautz-Morgan contrast effect are listed.

The data permit an improvement in the statistics of the Hubble diagram to $z=0.28$ and a new determination of the dependence of the absolute magnitude of the brightest cluster galaxy on cluster richness. A small correlation with richness is found whose amplitude is $0.20 \pm 0.18$ mag over all Abell richness classes $0-4$. As in previous studies, the shallowness of the correlation requires that if $M(1)$ is governed by a general luminosity function, $\phi(M)$, for fainter members, then the slope of $\phi(M)$ at $M(1)$ must be very steep.

The 16 new clusters with photometry have a dispersion in $M(1)$ of 0.26 mag. The dispersion of fully corrected magnitudes for the 65 clusters in the total sample is $0.276 \mathrm{mag}$.

The new points in the Hubble diagram give no indication that the $(m, z)$ relation deviates from the $q_{0}($ formal $)=+1$ line (i.e. with no evolutionary correction applied), nor that $3 \mathrm{C} 295$ is abnormally bright. The need for more data with $z>0.3$ is stressed.
\end{abstract}

Subject headings: cosmology - galaxies: clusters of - galaxies: redshifts

\section{INTRODUCTION}

Absorption-line redshifts of faint galaxies can now be measured routinely with the Hale $5 \mathrm{~m}$ reflector, using a new sky-subtraction prism spectrograph with an SIT television detector. The system and its first results were described by Westphal, Kristian, and Sandage (1975; hereafter WKS). We have started a program, using this instrument, to extend the Hubble diagram beyond the photographic cutoff at $z \approx 0.2$. The initial effort has been mainly on the bright end of this range, $z=0.2-0.3$, so as to gain experience with the system and test the redshift limits of various cluster surveys.

The present paper gives the results obtained to 1975 June on clusters which are not known radio sources ( $\S$ II). Most of these are clusters at the largest distance (range 6) of the Abell (1958) catalog. Other clusters are from special fainter surveys. A modest improvement in the statistics of the Hubble diagram to $z=0.3$ is achieved ( $(\mathrm{IV}$ ) by combining the redshift data with new $B V R$ broad-band photometry ( $\$$ III) for 16 of the clusters. New information on the absolute magnitude of first-ranked galaxies as a function of cluster richness has also been derived from the material (§ IV).

\section{NEW REDSHIFTS}

\section{a) Sample}

To improve our knowledge of the variation of absolute magnitude of first-ranked cluster galaxies with cluster richness, we plan to measure substantially all Abell clusters of richness class $R \geq 3$ (distance ranges 4,5 , and 6 ) as well as a number of sparser clusters in the same distance interval. Correlation of the absolute magnitude residuals with richness over a small redshift range gives the $M=f\left(N_{c}\right)$ relation, independent of assumptions about $q_{0}$.

In addition to these relatively bright Abell clusters, two sources of fainter clusters are available. Beginning in 1952, a search for clusters at the limit of the red Sky Survey plates was undertaken by Humason and Sandage (1957; hereafter HS) to find clusters more distant than Hydra $(0855+0321)$. Direct plates of the candidates were taken with the Hale reflector between 1952 and 1957 to determine if the most distant suspects were real. Many proved to be, and Humason (1957) attempted redshift measurements for a number of them, but was unsuccessful due to night-sky contamination. Later, redshifts for two of them $(0024+1654$ and $1447+2617)$ were measured by Baum (1962) from the shift of the continuum energy distribution. Baum's measurements were repeated by Oke (1971), using the multichannel scanner. Nine clusters found in this early survey are included in the present list, identified by HS in column (2) of Table 1.

More recently, we have begun a systematic survey for more distant clusters in the north and south galactic polar regions using the Palomar Schmidt, first with Eastman IIIaJ plates with a Wratten 4 filter, and now using the new fine-grain red 127-04 
TABLE 1

REDSHIFTS DETERMINED FROM OCTOBER 1974 TO JUNE 1975 USING THE DIGITAL SUBTRACTION

SPECTROGRAPH WITH SIT DETECTOR (HALE 5-METER REFLECTOR)

\begin{tabular}{|c|c|c|c|c|c|c|c|c|c|}
\hline $\begin{array}{c}\text { CLUSTER } \\
\text { (1) }\end{array}$ & $\begin{array}{l}\text { SOURCE } \\
\text { (2) }\end{array}$ & $\begin{array}{c}\alpha 1950 \\
(3)\end{array}$ & $\begin{array}{c}\delta 1950 \\
(4)\end{array}$ & $\begin{array}{l}b^{I I} \\
(5)\end{array}$ & $\begin{array}{c}z \\
(6)\end{array}$ & $\begin{array}{c}\text { Quality } \\
\text { (7) }\end{array}$ & $\begin{array}{c}\mathrm{R} \\
(8)\end{array}$ & $\begin{array}{l}\mathrm{BM} \\
(9)\end{array}$ & $\begin{array}{l}\text { NOTES } \\
(10)\end{array}$ \\
\hline $0024+1654$ & HS & 002400 & +165324 & $-45^{\circ}$ & 0.391 & $\mathrm{G}$ & (2) & III & WKS \\
\hline A 98 & ... & 004350.9 & +201153 & -42 & 0.1036 & $\mathrm{E}$ & 3 & II - III & WKS \\
\hline A 222 & $\mathrm{HS}$ & 013506.0 & $-13 \quad 14 \quad 48$ & -72 & 0.217 & $\mathrm{E}$ & 3 & II - III & WKS \\
\hline A 223 A & HS & 013535.0 & -130034 & -72 & 0.206 & $\mathrm{E}$ & 3 & II - III & WKS \\
\hline A $223 \mathrm{~B}$ & HS & 013528.4 & -130419 & -72 & 0.208 & $\mathrm{E}$ & & $\ldots$ & $\Delta \mathrm{V}_{\mathrm{r}} \sim 500 \mathrm{k} / \mathrm{s}$ \\
\hline A 223 (FG) & $\ldots$ & 013537.4 & -125955 & -72 & 0.049 & $\mathrm{E}$ & & $\ldots$ & Foreground \\
\hline A 278 & $\ldots$ & 015422.4 & +320015 & -29 & 0.0885 & $\mathrm{E}$ & 0 & $\ldots$ & WKS \\
\hline $0221-0849$ & HS(A348) & 022131.7 & -084914 & -61 & 0.274 & G & 1 & $\ldots$ & WKS \\
\hline A 376 & $\ldots$ & 024258.6 & +364150 & -20 & 0.0485 & $E$ & 0 & $\ldots$ & WKS \\
\hline A 520 & ... & 045127 & +024842 & -24 & 0.203 & $\mathrm{E}$ & 3 & III & WKS \\
\hline A 545 & ... & 053007 & -113502 & -23 & 0.154 & $\mathrm{E}$ & 4 & III & Obscured? \\
\hline A 586 & $\ldots$ & 072908.6 & +314422 & +22 & 0.172 & $\mathrm{E}$ & 3 & I - II & \\
\hline A 593 & $\ldots$ & 074601.9 & +725436 & +30 & 0.226 & G & 3 & II - III & WKS \\
\hline A 639 & $\ldots$ & $08 \quad 1504.2$ & +680405 & +33 & $0.291:$ & $\mathrm{P}$ & 3 & II - III & Should be \\
\hline A 655 & ... & 082212 & +4717 & +35 & 0.1245 & $E$ & 3 & $\mathrm{I}$ & confirmed \\
\hline $0851+6519$ & $3 \mathrm{aJ}(\mathrm{C} 6)$ & 085101.4 & +651914 & +37 & 0.283 & $\mathrm{E}$ & & I - II & \\
\hline A 777 & ... & 092327.0 & +783028 & +34 & 0.226 & $P$ & 4 & II - III & $z \pm 0.005$ \\
\hline A 795 & $\ldots$ & 092121.3 & +142328 & +40 & 0.1361 & $\mathrm{E}$ & 3 & II - III & WKS \\
\hline $0939+0912$ & HS(A 854) & 093920.9 & +091200 & +42 & 0.2075 & $\mathrm{E}$ & 1 & II - III & WKS \\
\hline A 963 & $\ldots$ & $1014 \quad 05.8$ & +391739 & +56 & 0.207 & $\mathrm{E}$ & 3 & II & WKS \\
\hline $1044+0918(\mathrm{FG})$ & HS & $1044 \quad 18.2$ & +091937 & +56 & 0.0908 & $\mathrm{E}$ & & $\ldots$ & Foreground \\
\hline $1044+0918$ & HS(A 1093) & $1044 \quad 10.9$ & +091853 & +56 & 0.226 & $\mathrm{E}$ & 1 & II - III & A 1093 \\
\hline A 1146 & $\ldots$ & 105847.1 & -222734 & +34 & 0.137 & $\mathrm{P}$ & 4 & I - II & WKS \\
\hline A 1514 & $\ldots$ & 121524.4 & +205700 & +80 & 0.200 & $\mathrm{G}$ & 3 & II - III & \\
\hline A 1550 & $\ldots$ & 122644.6 & +475800 & +69 & 0.254 & $\mathrm{E}$ & 3 & II - III & \\
\hline A 1758 & ... & 133038.7 & +504901 & +65 & 0.280 & $\mathrm{E}$ & 3 & III & \\
\hline A $1759 / 60$ & $\ldots$ & 133140.3 & +203053 & +78 & $0.168:$ & $\mathrm{P}$ & 3 & III & Not confirmed \\
\hline $1411+29$ & HS (A1878) & 141039.3 & +292830 & +72 & 0.222 & G & 1 & (I - II ): & Foreground? \\
\hline $1411+29$ & •. & 141039.6 & +292813 & +72 & 0.254 & $\mathrm{P}$ & & (I - II ): & \\
\hline $1438+2850$ & 3aJ (C5) & 143853.2 & +285000 & +66 & 0.248 & $\mathrm{E}$ & 2 & III & \\
\hline $1438+2850$ & $=\mathrm{A} 1952$ & 143853.7 & +285000 & +66 & 0.247 & $\mathrm{E}$ & & III & $\Delta \mathrm{V}_{\mathrm{r}} \sim 100 \mathrm{k} / \mathrm{s}$ \\
\hline $1438+2850(\mathrm{FG})$ & & 143856.2 & +285012 & +66 & 0.141 & $\mathrm{E}$ & & $\ldots$ & Foreground \\
\hline A 1957 & $\cdots$ & 144110.9 & +312324 & +65 & 0.241 & G & 3 & (I - II): & \\
\hline A 1961 & .. & $1442 \quad 08.4$ & +312811 & +65 & 0.234 & $\mathrm{E}$ & 3 & II - II & \\
\hline A 2111 & $\ldots$ & 153743.5 & +343512 & +53 & 0.228 & $\mathrm{E}$ & 3 & III & \\
\hline $\begin{array}{l}\text { A } 2125 \\
1613+3104\end{array}$ & $\ddot{3 a ̣ ̂}(\mathrm{C} 7)$ & $\begin{array}{lll}15 & 40 & 47.9 \\
16 & 13 & 49.6\end{array}$ & $\begin{array}{l}+662541 \\
+310457\end{array}$ & $\begin{array}{l}+43 \\
+46\end{array}$ & $\begin{array}{l}0.2465 \\
0.415\end{array}$ & $\begin{array}{l}\mathrm{E} \\
\mathrm{E}\end{array}$ & 4 & II - III & \\
\hline
\end{tabular}

experimental emulsion. Many remote clusters have been found, and three of the brighter ones from the IIIaJ survey are included in the redshifts reported here.

\section{b) Data}

The redshifts were determined with the spectrograph in the two-prism mode, which gives a spectral resolution (including detector) of $7 \AA$ at a dispersion of $125 \AA \mathrm{mm}^{-1}$ at $\mathrm{H} \gamma$. The measurements were made by cross-correlating the sky-subtracted spectrum of the unknown galaxy with a standard spectrum of the center of M31, shifted and degraded in resolution to match the cluster in question, as described by WKS (1975).

The results for 37 galaxies in or near 31 clusters are listed in Table 1. The cluster name (column [1]) is either the Abell number, designated as A, or an HS 1952 survey cluster designated in standard notation by the 1950 right ascension and declination, or a IIIaJ survey cluster, named the same way. Column (2) is the survey source, if it is not an Abell cluster. Columns (3) and (4) are the coordinates of the observed galaxy as estimated from transparent overlays placed over photographs. The accuracy of the coordinates is generally better than 15 seconds of arc. Column (5) gives the galactic latitude. The measured redshift and its quality (excellent, good, fair, poor) are listed in columns (6) and (7). The richness class for the Abell clusters are given in column (8), and the BautzMorgan cluster type in column (9). These types were either taken from Sandage and Hardy (1973, Table 1) or were estimated by us from prints. Some of the redshifts have been previously published in WKS, as 
noted in column (10), and are included here for convenience.

The accuracy of the tabulated redshifts is estimated to be about $\Delta z= \pm 0.001$. Several redshifts which were measured more than once reproduce to this accuracy. The listed redshifts have not been corrected for galactic rotation, since the correction is at most of the order of our accuracy, and in any case is negligible in the present context. The three foreground galaxies $(\mathrm{A} 223,1044+0918,1438+2850)$ were expected to be foreground objects from their appearance on direct photographs.

The redshifts listed for the two galaxies in the cluster $1411+29$ (A1878) differ by almost $10,000 \mathrm{~km} \mathrm{~s}^{-1}$. Although neither spectrum is of the best quality and one is rather poor, there is clearly a large velocity

TABLE 2

PHOTOMETRY OF 16 CLUSTERS THAT HAVE NEW REDSHIFTS

\begin{tabular}{|c|c|c|c|c|c|c|c|c|c|c|c|c|}
\hline $\begin{array}{l}\text { CLUSTER } \\
\text { (1) }\end{array}$ & $\begin{array}{l}\text { OBJECT } \\
\text { (2) }\end{array}$ & $\begin{array}{l}z \\
(3)\end{array}$ & $\begin{array}{l}b^{\text {II }} \\
(4)\end{array}$ & $\begin{array}{l}\text { ap } \\
(5)\end{array}$ & $\begin{array}{c}\mathrm{V} \\
\mathrm{mag} \\
(6)\end{array}$ & $\begin{array}{c}\text { B } \\
\mathrm{mag} \\
(7)\end{array}$ & $\begin{array}{c}\mathrm{R} \\
\mathrm{mag} \\
(8)\end{array}$ & $\begin{array}{l}B-V \\
\text { mag } \\
(9)\end{array}$ & $\begin{array}{c}\mathrm{V}-\mathrm{R} \\
\mathrm{mag} \\
(10)\end{array}$ & $\begin{array}{l}\mathrm{v}_{\mathrm{SM}} \\
\mathrm{mag} \\
(11)\end{array}$ & $\begin{array}{c}\mathrm{V}_{\mathrm{SM}}-\mathrm{A}_{\mathrm{V}}-\mathrm{K}_{\mathrm{v}} \\
\mathrm{mag} \\
(12)\end{array}$ & (13) \\
\hline A 586 & G1 & 0.172 & $+22^{\circ}$ & $\begin{array}{l}12 . .2 \\
18.8\end{array}$ & $\begin{array}{l}17.26^{ \pm} .022 \\
16.74 \pm .020\end{array}$ & $\begin{array}{c}18.67 \pm .035 \\
\cdots\end{array}$ & $\begin{array}{l}16.19 \pm .027 \\
15.69 \pm .021\end{array}$ & $\begin{array}{c}1.41 \pm .041 \\
\ldots .\end{array}$ & $\begin{array}{l}1.07 \pm .035 \\
1.05 \pm .028\end{array}$ & 16.85 & 16.38 & 4.713 \\
\hline A 593 & G1 & 0.226 & +30 & $\begin{array}{r}7.6 \\
12.2 \\
18.8\end{array}$ & $\begin{array}{c}\ldots . .0 \\
18.20^{ \pm} .023 \\
18.06^{ \pm} .037\end{array}$ & $\begin{array}{c}19.97 \pm .061 \\
\ldots\end{array}$ & $\begin{array}{l}17.51 \pm .028 \\
17.10 \pm .017 \\
16.99 \pm .017\end{array}$ & $\begin{array}{c}\ldots . . \\
1.77 \pm .065 \\
\ldots .\end{array}$ & $\begin{array}{l}1.10 \pm .029 \\
1.07 \pm .041\end{array}$ & 18.04 & 17.46 & 4. 831 \\
\hline A 639 & G1 & $0.291:$ & +33 & 12.2 & $18.40^{ \pm} .037$ & $\cdots$ & $\ldots$. & $\cdots$ & $\cdots \cdot$ & 18.30 & 17.48 & 4.941 \\
\hline $\begin{array}{l}\text { A } 777 \\
\Delta V(1-B)=0.2 \mathrm{mag} .\end{array}$ & $\begin{array}{l}\mathrm{G} 1+\mathrm{A} \\
\mathrm{B}\end{array}$ & 0.226 & $\begin{array}{l}+34 \\
+34\end{array}$ & 18.8 & $\begin{array}{l}17.49^{ \pm} .022 \\
18.84^{ \pm} .032\end{array}$ & $\begin{array}{c}19.27^{ \pm} .048 \\
\ldots .\end{array}$ & $\begin{array}{l}16.50 \pm .022 \\
17.72 \pm .030\end{array}$ & $\begin{array}{c}1.78^{ \pm} .053 \\
\ldots .\end{array}$ & $\begin{array}{l}0.99 \pm .031 \\
1.12 \pm .044\end{array}$ & $\begin{array}{l}17.49 \\
18.68\end{array}$ & $\begin{array}{l}17.49(\mathrm{G} 1) \\
18.12(\mathrm{~B})\end{array}$ & 4.831 \\
\hline A 795 & G1 & 0.1361 & +40 & 12.2 & $16.89 \pm .013$ & $18.20^{ \pm} .019$ & $15.90 \pm .019$ & $1.31 \pm .023$ & $0.99 \pm .023$ & 16.57 & 16.29 & 4.610 \\
\hline $\begin{array}{l}\text { A } 963 \\
\text { Double } \\
\Delta m=1.25\end{array}$ & $\mathrm{G} 1+\mathrm{G} 2$ & 0.207 & +56 & $\begin{array}{l}12.2 \\
18.8\end{array}$ & $\begin{array}{l}17.24 \pm .016 \\
17.07 \pm .008\end{array}$ & $\begin{array}{l}18.92 \pm .019 \\
18.79 \pm .026\end{array}$ & $\begin{array}{l}16.14 \pm .008 \\
16.01 \pm .036\end{array}$ & $\begin{array}{l}1.68 \pm .025 \\
1.72 \pm .027\end{array}$ & $\begin{array}{l}1.10 \pm .018 \\
1.06 \pm .037\end{array}$ & 17.36 & 16.97 & 4.791 \\
\hline A 1146 & G1 & 0.137 & +34 & $\begin{array}{l}12.2 \\
18.8\end{array}$ & $\begin{array}{l}16.59 \pm .015 \\
16.18 \pm .009\end{array}$ & $\begin{array}{l}18.22 \pm .026 \\
17.63 \quad .023\end{array}$ & $\begin{array}{l}15.61 \pm .015 \\
15.23 \pm .028\end{array}$ & $\begin{array}{l}1.63 \pm .030 \\
1.45 \pm .025\end{array}$ & $\begin{array}{l}0.98 \pm .021 \\
0.95 \pm .029\end{array}$ & 16.20 & 15.98 & 4.614 \\
\hline A 1514 & G1 & 0.200 & +80 & $\begin{array}{l}12.2 \\
18.8\end{array}$ & $\begin{array}{l}17.71 \pm .010 \\
17.39 \pm .012\end{array}$ & $\begin{array}{l}19.34 \pm .034 \\
19.04 \pm .026\end{array}$ & $\begin{array}{l}16.67 \pm .009 \\
16.36 \pm .018\end{array}$ & $\begin{array}{l}1.63 \pm .035 \\
1.65 \pm .029\end{array}$ & $\begin{array}{l}1.04 \pm .013 \\
1.03 \pm .022\end{array}$ & 17.44 & 17.05 & 4.778 \\
\hline $\begin{array}{l}\text { A } 1550 \\
\text { A triple. One } \\
\text { dominant and two } \\
\text { small satellites }\end{array}$ & G1 & 0.254 & +69 & $\begin{array}{l}12.2 \\
18.8\end{array}$ & $\begin{array}{l}18.09 \pm .028 \\
17.86 \pm .025\end{array}$ & $\begin{array}{c}19.79 \pm .046 \\
\ldots .\end{array}$ & $\begin{array}{l}17.02 \pm .024 \\
16.79 \pm .028\end{array}$ & $\begin{array}{c}1.70 \pm .054 \\
\cdots\end{array}$ & $\begin{array}{l}1.07 \pm .037 \\
1.07 \pm .037\end{array}$ & 17.93 & 17.34 & 4.883 \\
\hline A 1758 & G1 & 0.280 & +65 & $\begin{array}{l}12.2 \\
18.8\end{array}$ & $\begin{array}{l}18.37 \pm .028 \\
18.12 \pm .020\end{array}$ & $\begin{array}{c}20.12 \pm .047 \\
\cdots \cdot\end{array}$ & $\begin{array}{l}17.31 \pm .038 \\
16.95 \pm .020\end{array}$ & $\begin{array}{c}1.75 \pm .055 \\
\cdots\end{array}$ & $\begin{array}{l}1.06 \pm .047 \\
1.17 \pm .028\end{array}$ & 18.21 & 17.51 & 4.926 \\
\hline A 1759 & G1 & $0.168:$ & +78 & $\begin{array}{l}12.2 \\
18.8\end{array}$ & $\begin{array}{l}17.58 \pm .017 \\
17.43 \pm .021\end{array}$ & $\begin{array}{l}19.19 \pm .018 \\
19.01 \pm .043\end{array}$ & $\begin{array}{l}16.57 \pm .013 \\
16.44 \pm .011\end{array}$ & $\begin{array}{l}1.61 \pm .024 \\
1.58 \pm .048\end{array}$ & $\begin{array}{l}1.01 \pm .021 \\
0.99 \pm .024\end{array}$ & 17.36 & 17.07 & 4.702 \\
\hline A 1957 & A & 0.241 & +65 & 12.2 & $18.30 \pm .040$ & $19.97 \pm .037$ & $17.16^{ \pm} .030$ & $1.67 \pm .054$ & $1.14 \pm .050$ & 18.16 & 17.63 & 4.859 \\
\hline A 1961 & G1 & 0.234 & +64 & 12.2 & $17.57 \pm .020$ & $19.26 \pm .034$ & $16.50 \pm .017$ & $1.69^{ \pm} .039$ & $1.07^{ \pm .026}$ & 17.43 & 16.91 & \\
\hline & G2 & $"$ & $"$ & $\begin{array}{l}12.2 \\
18.8\end{array}$ & $\begin{array}{l}17.72 \pm .022 \\
17.73 \pm .053\end{array}$ & $\begin{array}{c}19.46 \pm .037 \\
\cdots\end{array}$ & $\begin{array}{l}16.65 \pm .014 \\
16.55 \pm .033\end{array}$ & $\begin{array}{c}1.74 \pm .043 \\
\ldots .\end{array}$ & $\begin{array}{l}1.07 \pm .026 \\
1.18^{ \pm .062}\end{array}$ & 17.66 & 17.14 & 4.850 \\
\hline A 2111 & G1 & 0.228 & +53 & $\begin{array}{l}12.2 \\
18.8\end{array}$ & $\begin{array}{l}17.96^{ \pm} .012 \\
17.68^{ \pm} .020\end{array}$ & $\begin{array}{l}19.70 \pm .031 \\
19.37 \pm .032\end{array}$ & $\begin{array}{l}16.91^{ \pm} .013 \\
16.60^{ \pm} .024\end{array}$ & $\begin{array}{l}1.74 \pm .033 \\
1.69 \pm .038\end{array}$ & $\begin{array}{l}1.05 \pm .018 \\
1.08 \pm .031\end{array}$ & 17.76 & 17.27 & 4.835 \\
\hline $\begin{array}{l}\text { A } 2125 \\
\text { A is } 3 \text { galaxies }\end{array}$ & $\mathrm{A}$ & 0.2465 & +43 & $\begin{array}{l}18.8 \\
30.6\end{array}$ & $\begin{array}{l}16.84 \pm .016 \\
16.62 \pm .012\end{array}$ & $\begin{array}{c}18.51 \pm .038 \\
\ldots \ldots\end{array}$ & $\begin{array}{l}15.71 \pm .009 \\
15.61 \pm .022\end{array}$ & $\begin{array}{c}1.67 \pm .041 \\
\cdots\end{array}$ & $\begin{array}{l}1.13 \pm .018 \\
1.01 \pm .025 \\
\text { Single Bgts }\end{array}$ & 17.67 & 17.07 & \\
\hline & G3 & $"$ & $"$ & & $\begin{array}{c}17.93 \pm .026 \\
\cdots .\end{array}$ & $\begin{array}{c}19.78^{ \pm} .054 \\
\cdots . .\end{array}$ & $\begin{array}{l}16.90 \pm .012 \\
16.50 \pm .025\end{array}$ & $\begin{array}{c}1.85 \pm .060 \\
\ldots . .\end{array}$ & $\begin{array}{c}1.03 \pm .029 \\
\cdots\end{array}$ & 17.66 & 17.06 & 4.869 \\
\hline $0939+0912$ & $\mathrm{~K}$ & 0.2075 & +42 & $\begin{array}{l}12.2 \\
18.8\end{array}$ & $\begin{array}{l}17.82 \pm .016 \\
17.52:^{ \pm} .082\end{array}$ & $\begin{array}{c}19.35 \pm .031 \\
\ldots .\end{array}$ & $\begin{array}{l}16.75 \pm .013 \\
16.47 \pm .022\end{array}$ & $\begin{array}{c}1.53 \pm .035 \\
\cdots\end{array}$ & $\begin{array}{l}1.07 \pm .021 \\
1.05 \pm .085\end{array}$ & 17.60 & 17.15 & 4.791 \\
\hline
\end{tabular}


difference. The smaller redshift galaxy is centrally located in the cluster, and is its brightest member, but it is photometrically peculiar, and may be a foreground object. This cluster will be discussed further in a later paper of this series.

The geometry of A222-223 is interesting. There are three separate condensations of galaxies lying nearly on a straight line 15'.8 long. The entire configuration was listed by HS as a single field. The southwest condensation $(z=0.217)$ is listed by Abell as A222, while the northeast pair (separation $4{ }^{\prime} .2$ ) is apparently listed by Abell as a single cluster, A223. The galaxies listed as A223A and A223B in Table 1 are the brightest members of the northeast pair of condensations, and the similarity of their redshifts suggests that they are in fact at the same distance.

Abell (1958) gives positions for A1759 and A1760 which are only $2^{\prime} .4$ apart. Both are listed as richness 3 , with appreciably different magnitudes (17.6 and 17.2). We do not find a similar configuration at the listed position, but rather a very extended grouping $\left(\sim 10^{\prime}\right.$ in diameter) with no striking subcondensations.

Table 1 suggests that the redshift limit of the Abell catalog is just less than $z=0.3$. The HS survey clusters overlap the faint end of the Abell catalog and extend to larger redshifts $(z=0.39$ for $0024+1654)$. We do not yet know the limits of the IIIaJ and 127-04 surveys. The brightest of the clusters from these special surveys were selected to overlap the faint end of the Abell survey, and the redshifts of the three clusters listed $(z=0.252,0.283$, and 0.415$)$ indicate that this expectation is met. These clusters are well above the faint survey limit, and no serious attempt was made during these initial measurements to push to the faintness limits of the spectrograph.

\section{MAGNITUDES AND COLORS}

\section{a) Measurements}

Broad-band $B V R$ photometry for 16 of the 31 clusters of Table 1 was done using the same procedures, techniques, filters, photocell (S20, FW130), and corrections previously used for a sample of nearer clusters (Sandage 1972b, Paper II).

Most of the galaxies were measured with two apertures to provide an internal check on the aperture correction to a standard metric diameter. The diameters used, in arcsec, are listed in column (5) of Table 2. Columns (6)-(8) give the magnitudes through the corresponding apertures. The tabulated magnitude errors are rms values, calculated from variations of the net counts over many individual $10 \mathrm{~s}$ integrations. ${ }^{1}$

\footnotetext{
${ }^{1}$ A technical problem exists concerning the present photometry. Some of the galaxies are redder in $B-V$ than the standard stars observed on the nights in question, and an extrapolation of about $0.3 \mathrm{mag}$ was required in the transformations that relate $(b-v)_{\text {natural }}$ to $B-V$. The problem does not exist in $V-R$. The listed rms errors are from the photon statistics alone.
}

\section{b) Corrections for Aperture Effect, $K$-Dimming, and Galactic Absorption}

We have used the same aperture correction as for the nearer clusters (Sandage 1972a, Table 3). The aperture growth curve was adopted here as if $q_{0}=+1$, which requires the argument of the curve to be $\log \left[\theta z /(1+z)^{2}\right]$. The results, however, are not very sensitive to this assumption, since the standard diameter is very large $\left(86 \mathrm{kpc}\right.$ if $\left.H_{0}=50\right)$. At this diameter, the gradient of the growth curve is small, which ensures that the correction itself is nearly independent of $q_{0}$ because we are already close to an "asymptotic" magnitude. Stated differently, it does not much matter where we read the abscissa of the $\Delta m=f(\theta, z)$ function as long as $\theta$ is large enough so that the function is nearly flat. The difference in the aperture corrections between the $q_{0}=+1$ case used here and the $q_{0}=0$ case (abscissa of the growth curve $\left.=\log \left[\theta z(1+z / 2) /(1+z)^{2}\right]\right)$ is only 0.03 mag at $z=0.28$. Since

$$
m=5 \log c z+1.086\left(1-q_{0}\right) z+O\left(z^{2}\right)+C
$$

(Robertson 1955), it follows than an error of $0.03 \mathrm{mag}$ at $z=0.28$ corresponds to an error $\delta q=\delta m / 1.09 z \approx$ 0.10 , which is only 10 percent of the difference in the assumed values of $q_{0}$ between the two models.

The $V$ magnitudes, reduced to the standard metric size by the adopted precepts, and denoted as $V_{\mathrm{SM}}$, are listed in column (11) of Table 2. Corrections for galactic absorption (Sandage 1973, Paper V) and for $K$ dimming (Whitford 1971) have been applied in column (12).

\section{c) Extension of the Color-Redshift Relation}

The observed colors from columns (9) and (10) of Table 2 are plotted as open circles versus redshift in Figure 1. The error bars are the listed rms values. The data for nearer clusters are shown as closed circles, and are taken from previous measurements [Paper II, Table 1; Paper V, Table 6]. The solid lines are the expected $K_{B}-K_{V}$ reddening trajectories from the measurements of Whitford (1971), calculated by him from his absolute energy distributions. The curve for $V-R$ is taken from Table 5 of Paper V.

While the agreement between the observations and the predicted $V-R$ values is satisfactory to within systematic differences of $\sim \pm 0.03$ in the range $0<z<0.5$, it is not good in $B-V$. A systematic difference of $\sim 0.06 \mathrm{mag}$ exists for $z>0.2$; the observed values are redder than the prediction. At present, we feel that the effect may not be real, but that it may be due to a combination of the technical problem mentioned above (footnote $1, \S$ III $a$ ) and a possible breakdown of the defining equation (A7) of Matthews and Sandage (1963) that relates the natural $b-v$ system to standard $B-V$ colors. No calculations were made in that reference for such red stars, and special spectrum-scanner observations are required to rederive the necessary transformation equation for $B-V>1.6$. 


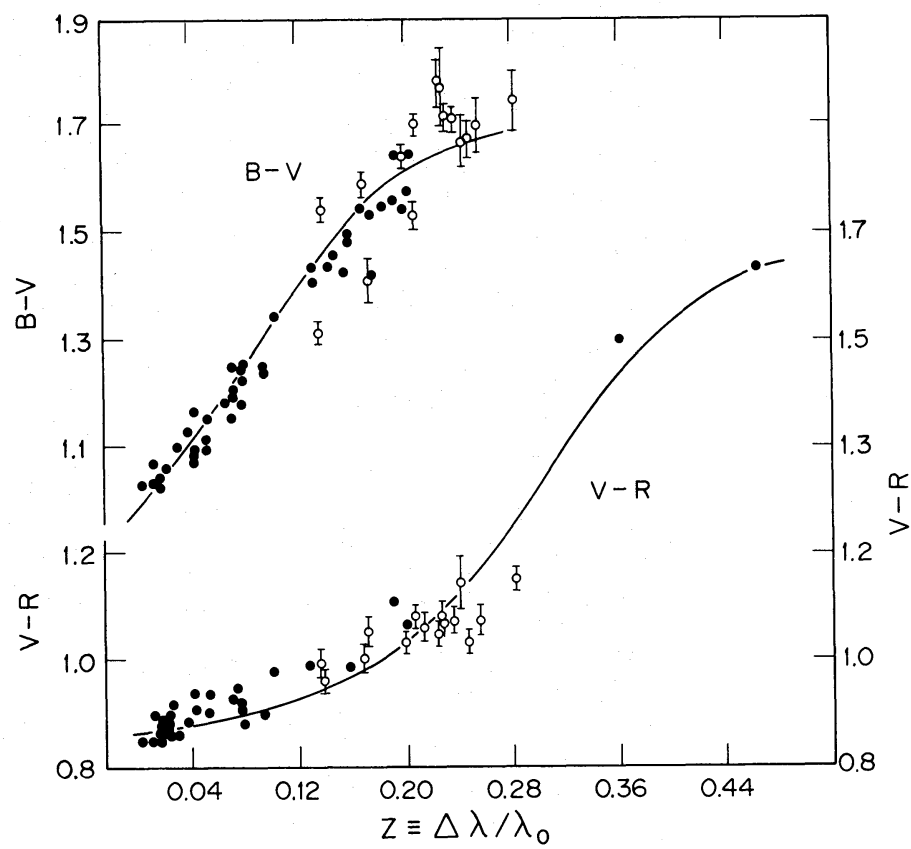

FIG. 1.-Observed colors of the brightest several E and S0 galaxies in clusters as a function of redshift. Open circles are the 16 new clusters with redshifts and photometry from Tables 1 and 2 . Closed circles are for clusters with previous data (Sandage 1972b, Table 1; Sandage 1973, Table 6). Solid lines are from Whitford's (1971) calculated $K$-corrections for a standard elliptical galaxy.

\section{RICHNESS CORRECTION}

The data for the 16 new clusters are plotted as closed circles in Figure 2, together with 50 clusters (open circles) from previous work. The corrections to the magnitudes are for aperture effect, $K$-dimming, and galactic absorption only. The line has a slope of 5 as required by a linear velocity-distance relation in empty Euclidean space, or by a Friedmann model with $q_{0}=+1$. (We do not mean to suggest that either of these models is true: the line is drawn only for convenience.) Its equation is $V_{C} \equiv V_{\mathrm{SM}}-K_{V}-A_{V}=$ $5 \log c z-6.803$, where the constant has been adjusted for a zero mean residual for the open circles.

Two noteworthy features of Figure 2 are (1) the small dispersion in $M_{v}{ }^{c}$ of the 16 new points alone $(\sigma=0.26 \mathrm{mag})$, showing again the remarkably well defined mean absolute magnitude of the first-ranked cluster galaxy, and (2) the slight offset toward brighter magnitudes of the new data compared with the old. The difference amounts to $M_{v}{ }^{c}(1)=0.139 \pm 0.081 \mathrm{mag}$ which, although small, seems real. We believe that this difference is due to a weak correlation between $M$ (brightest) and cluster richness, since there is a strong correlation between richness and redshift in our data. In the redshift range $0<z<0.12$, the mean richness class is $\langle R\rangle=0.6$, with all clusters in the range $0 \leq R \leq 2$. For redshifts $0.12<z<0.22$ the mean richness is $\langle R\rangle=2.4$. For $0.22<z<0.30$ (all new clusters), $\langle R\rangle=3.3$.

Ideally, to sort out the richness correction from FIG. 2.-The Hubble diagram for first-ranked members of 50 previously measured clusters (open circles), and for the 16 new clusters with photometry (closed circles). The magnitudes have been corrected for aperture effect, $K$-dimming, and galactic absorption. The line has the equation $V_{c}=5 \log c z-$ 6.803. The mean dispersion of the absolute magnitude of the first-ranked galaxy is 0.305 mag. possible time-dependent effects such as deceleration and evolution one should examine the correction at each redshift from coeval data. To do this would 
require extensive material, uniformly distributed in richness, over each small redshift interval. Such data do not now exist, and we have adopted a crude first approximation which seems to be all that can be supported by the data in hand. The principal change from earlier work is a substantial increase in the number of clusters with $R=3$ and 4 .

The data were split into the two redshift intervals $0<z<0.10$ and $0.13<z<0.26$, and within each interval a mean value of the quantity $5 \log c z-V_{c}$ was computed separately for the clusters in each richness class. The assumptions underlying this procedure are that $M(1)$ for each richness class has a well-defined mean magnitude over a given redshift range, and that the function $M(\log c z)$ is adequately represented over the redshift range by a straight line of slope 5. Since the correction is so small (see below), these assumptions appear to be justified a posteriori for the present data.

The results are given in Table 3 . Within each redshift range, there appears to be a small but systematic correlation between $R$ and $\left\langle 5 \log c z-V_{c}\right\rangle$. Although the distribution of richness in the two redshift intervals is quite different, there is an overlap in richness classes 1 and 2 . The values of $\left\langle 5 \log c z-V_{c}\right\rangle$ are not well determined, but they agree within the errors between the two redshift ranges, and we have adopted the simplifying assumption that there is a single correction over the entire redshift range considered. The values are given in the last part of Table 3 .

The values of $\left\langle 5 \log c z-V_{c}\right\rangle$ with their rms errors are plotted in Figure 3, together with the older determinations (open squares) from Sandage and Hardy (1973, Table 4). Shown along the right-hand ordinate, and listed in the penultimate column of Table 3

\section{TABLE 3}

Deviations from the Mean Hubble Line as a Function of RICHNESS IN Two REDSHIFT RANGES

\begin{tabular}{|c|c|c|c|}
\hline$R$ & $\left\langle 5 \log c z-V_{c}\right\rangle$ & $n$ & $\sigma\left(M_{v}^{c}\right)(\mathrm{mag})$ \\
\hline \multicolumn{4}{|c|}{$0<z<0.10$} \\
\hline $\begin{array}{l}0 \ldots \ldots \\
1 \ldots \ldots \\
2 \ldots \ldots\end{array}$ & $\begin{array}{l}6.746 \pm 0.074 \\
6.793 \pm 0.058 \\
6.837 \pm 0.148\end{array}$ & $\begin{array}{r}18 \\
8 \\
6\end{array}$ & $\begin{array}{l}0.303 \\
0.154 \\
0.332\end{array}$ \\
\hline \multicolumn{4}{|c|}{$0.13<z<0.26$} \\
\hline $\begin{array}{l}1 \ldots \ldots \\
2 \ldots \ldots \\
3 \ldots \ldots \\
4 \ldots \ldots\end{array}$ & $\begin{array}{l}6.693 \pm 0.186 \\
6.834 \pm 0.128 \\
6.903 \pm 0.084 \\
6.923 \pm 0.178\end{array}$ & $\begin{array}{r}5 \\
5 \\
15 \\
4\end{array}$ & $\begin{array}{l}0.373 \\
0.257 \\
0.315 \\
0.308\end{array}$ \\
\hline$R$ & $\stackrel{\text { Mean }}{\left\langle 5 \log c z-V_{c}\right\rangle}$ & $\begin{array}{l}\text { Adopt } \\
\left\langle\Delta M_{v}\right\rangle\end{array}$ & $n$ \\
\hline $\begin{array}{l}0 \ldots \ldots \\
1 \ldots \ldots \\
2 \ldots \ldots \\
3 \ldots \ldots \\
4 \ldots \ldots\end{array}$ & $\begin{array}{l}6.746 \pm 0.074 \\
6.755 \pm 0.072 \\
6.835 \pm 0.090 \\
6.903 \pm 0.084 \\
6.923 \pm 0.178\end{array}$ & $\begin{array}{l}-0.07 \\
-0.02 \\
+0.02 \\
+0.07 \\
+0.12\end{array}$ & $\begin{array}{r}18 \\
13 \\
11 \\
15 \\
4\end{array}$ \\
\hline
\end{tabular}

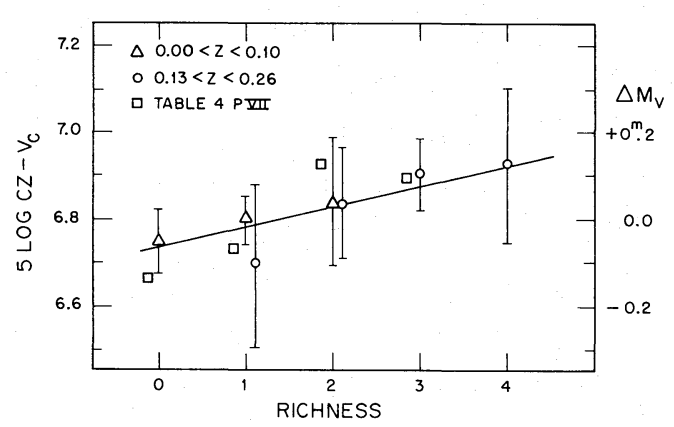

FIG. 3.-Derived dependence of $M(1)$ on cluster richness in two redshift ranges. The dependence derived earlier (Sandage and Hardy 1973, Table 4) is shown by open squares.

(bottom), are the values of the correction, $\left\langle M_{v}\right\rangle$, normalized to zero between richness class 1 and 2 .

The most striking feature of the richness correction is that it is so small. This near independence of the brightness of the brightest member on the total membership of the cluster is also known to extend to groups with less than 10 members (Sandage 1975, Paper VIII), and this requires that the integrated luminosity function $\phi(M)$ for cluster galaxies be nearly vertical at the bright end, if the absolute magnitude of the first-ranked member is governed by the luminosity distribution of the fainter members. It can be shown that the slope of $\phi(M)$ at $M(1)$ must be $d \log \phi(M) / d M \geq 5$ to explain the data of Figure 3 in this way.

\section{CORRECTED HUBBLE DIAGRAM}

\section{a) Data}

From the discussion in $\S I V$, it is clear that the richness correction derived there will largely remove the "excess" brightness of the 16 new clusters. But the argument still seems somewhat tautological, and it will be desirable to reexamine the question with more extensive material in the future. Since there are very few nearby rich clusters $(R \geq 3)$, the new data will have to be for distant sparse clusters.

In addition to the corrections discussed in $\S$ III and $\S I V$, a further correction for Bautz-Morgan (BM) cluster type has been applied ${ }^{2}$ to obtain the data in Table 4.

The columns of Table 4 are mostly self-explanatory. The richness class in column (4) is from the Abell catalog except for $0024+1654$, which was estimated by us. As before, $V_{c}$ magnitudes in column (6) are corrected only for aperture effect, $K$-dimming, and galactic absorption; the $V_{c}{ }^{R, \mathrm{BM}}$ values in column (7) are additionally corrected for richness and BautzMorgan effects.

The data in Table 4 are plotted in Figure 4. The equation of the line is

$$
V_{c}{ }^{R, \mathrm{BM}}=5 \log c z-6.865 .
$$

${ }^{2}$ Note the typographical error in $\Delta M_{v}{ }^{c}(1)$ for BM type II-III in Table 3 of Sandage and Hardy (1973). The entry should read +0.05 . All calculations here and in that paper were, however, made with the correct value: the error is in the table only. 
TABLE 4

SUMMARY OF PARAMETERS FOR 66 CLUSTERS WHICH HAVE FIRST CLASS DATA

\begin{tabular}{|c|c|c|c|c|c|c|c|}
\hline $\begin{array}{l}\text { CLUSTER } \\
\text { (1) }\end{array}$ & $\begin{array}{c}\text { ABELL } \\
\text { (2) }\end{array}$ & $\begin{array}{c}z \\
(3)\end{array}$ & $\begin{array}{c}R \\
(4)\end{array}$ & $\begin{array}{l}\mathrm{BM} \\
(5)\end{array}$ & $\begin{array}{l}\mathrm{V}_{C} \\
(6)\end{array}$ & $\mathrm{V}_{C_{(7)}^{R, B M}}^{R}$ & $\begin{array}{c}\log c z \\
(8)\end{array}$ \\
\hline \multicolumn{8}{|c|}{ FROM TABLE 2 OF PAPER II } \\
\hline VIRGO & ... & 0.00379 & (1) & III & 8.45 & 8.21 & 3.056 \\
\hline FORNAX & ... & 0.00509 & (1) & II - III & 8.90 & 8.83 & 3. 184 \\
\hline PEG I & ... & 0.0128 & $(0)$ & II & 11.20 & 11.15 & 3.584 \\
\hline $0122+3305$ & $\ldots$ & 0.0170 & (1) & II & 11.70 & 11.70 & 3.710 \\
\hline PERSEUS & A 426 & 0.0181 & 2 & II - III & 11.54 & 11.51. & 3.735 \\
\hline COMA & A 1656 & 0.0222 & 2 & II & (11.51) & 11.55 & 3.824 \\
\hline HERCULES & A 2151 & 0.0341 & 2 & III & 13.12 & 12.92 & 4.017 \\
\hline $2308+0720$ & ... & 0.0428 & (0) & II - III & 13.57 & 13.45 & 4.109 \\
\hline $2322+1425$ & A 2589 & 0.0440 & 0 & I & 14.08 & 14.37 & 4.120 \\
\hline $1145+5559$ & A 1377 & 0.0516 & 1 & II - III & 14.39 & 14.32 & 4.191 \\
\hline $0106-1536$ & A 151 & 0.0526 & 1 & II & 14.03 & 14.03 & 4.198 \\
\hline $1024+1039$ & A 1020 & 0.0649 & 1 & III & 14.81 & 14.57 & 4.290 \\
\hline $1239+1852$ & A 1589 & 0.0718 & 0 & II - III & 14.52 & 14.40 & 4.333 \\
\hline $1520+2754$ & A 2065 & 0.0722 & 2 & ШI & 15.24 & 15.04 & 4.334 \\
\hline $0705+3506$ & A 568 & 0.0779 & 0 & II - III & 15.15 & 15.03 & 4.369 \\
\hline $1513+0433$ & A 2048 & 0.0944 & 1 & IIII & 15.50 & 15.26 & 4.452 \\
\hline $1431+3146$ & A 1930 & 0.1312 & 1 & III & 16.06 & 15.82 & 4.595 \\
\hline $1055+5702$ & A 1132 & 0.1345 & 1 & II - Ш & 15.93 & 15.86 & 4.606 \\
\hline $1153+2341$ & A 1413 & 0.1426 & 3 & I & 15.46 & 15.89 & 4.631 \\
\hline $1641+1327$ & A 2224 & 0.1499 & 3 & III & 16.61 & 16.46 & 4.652 \\
\hline $1534+3749$ & A 2100 & 0.1532 & 3 & III & 16.55 & 16.40 & 4.662 \\
\hline $0025+2223$ & A 31 & 0.1594 & 2 & III & 16.58 & 16.38 & 4.680 \\
\hline $1228+1050$ & A 1553 & 0.1651 & 2 & II - III & 16.90 & 16.87 & 4.695 \\
\hline $0138+1832$ & A 234 & 0.1730 & 1 & II & 17.17 & 17.17 & 4.714 \\
\hline $1309-0105$ & A 1689 & 0.1745 & 4 & I - II & 16.93 & 17.18 & 4.719 \\
\hline $1304+3110$ & A 1677 & 0.1831 & 2 & III & 16.90 & 16.70 & 4.740 \\
\hline $0925+2044$ & A 801 & 0.1917 & 2 & I - II & 16.54 & 16.69 & 4.760 \\
\hline $1253+4422$ & A 1643 & 0.1979 & 1 & ШII & 17.66 & 17.42 & 4.774 \\
\hline $0855+0321$ & A 732 & 0.2018 & 1 & II - III & 17.07 & 17.00 & 4.782 \\
\hline $1447+2617$ & ... & 0.36 & (2) & II - III & 18.77 & 18.74 & 5.033 \\
\hline $0024+1654$ & $\ldots$ & 0.392 & (2) & III & 18.34 & 18.14 & 5.057 \\
\hline \multicolumn{8}{|c|}{ 3C RADIO CLUSTERS } \\
\hline $3 \mathrm{C} 31$ & ... & 0.0169 & $(0)$ & ш & 11.90 & 11.61 & 3.706 \\
\hline 3C 40 & A 194 & 0.0180 & 0 & II & 12.24 & 12.19 & 3.732 \\
\hline 3C 66 & A 347 & 0.0215 & 0 & II - WI & 12.69 & 12.57 & 3.810 \\
\hline $3 \mathrm{C} 465$ & A 2634 & 0.0301 & 1 & I - II & 13.06 & 13.17 & 3.956 \\
\hline $3 C 338$ & A 2199 & 0.0303 & 2 & I & 12.53 & 12.91 & 3.958 \\
\hline $3 \mathrm{C} 317$ & A 2052 & 0.0351 & 0 & II & 13.38 & 13.33 & 4.022 \\
\hline M23-112 & (A 2638) & 0.0825 & 2 & III & 15.33 & 15.13 & 4.394 \\
\hline 3C 219 & $\ldots$ & 0.1745 & (2) & II & 16.88 & 16.92 & 4.719 \\
\hline $3 \mathrm{C} 28$ & A 115 & 0.1959 & 3 & III & 17.10 & 16.95 & 4.769 \\
\hline 3C 295 & $\ldots$ & 0.461 & $(1)$ & 1 & 18.63 & 18.97 & 5.141 \\
\hline \multicolumn{8}{|c|}{ WESTERLUND AND WALL (SOME ARE HMS GROUPS) } \\
\hline $0036+03$ & ... & 0.0145 & 0 & III & 12.11 & 11.82 & 3.697 \\
\hline $0131-36$ & ... & 0.0298 & 0 & III & 13.19 & 12.90 & 3.951 \\
\hline $0153+05$ & ... & 0.0188 & 0 & II & 11.98 & 11.93 & 3.751 \\
\hline $0915-11$ & ... & 0.0522 & 0 & I & 13.58 & 13.87 & 4. 194 \\
\hline $1245-41$ & ... & 0.0113 & 2 & $\amalg$ & 10.90 & 10.94 & 3.498 \\
\hline $1332-33$ & ... & 0.0114 & 0 & I & 10.87 & 11.16 & 3.535 \\
\hline $1400-33$ & ... & 0.0138 & 0 & I & 11.01 & 11.30 & 3.617 \\
\hline $2152-69$ & $\ldots$ & 0.0266 & 0 & ... & 13.19 & $\ldots$ & 3.902 \\
\hline$\underline{2247+11}$ & $\ldots$ & 0.0268 & 0 & III & 12.40 & 12.11 & 3.906 \\
\hline \multicolumn{8}{|c|}{ THE 16 NEW CLUSTERS HERE } \\
\hline $0729+3144$ & A 586 & 0.172 & 3 & I - II & 16.38 & 16.58 & 4.713 \\
\hline $0746+7254$ & A 593 & 0.226 & 3 & II - III & 17.46 & 17.49 & 4.831 \\
\hline $0815+6804$ & A 639 & $0.291 ?$ & 3 & II - II & 17.48 & 17.50 & 4.941 \\
\hline $0923+7830$ & A 777 & $0.226 ?$ & 4 & II - III & 17.49 & 17.56 & 4.831 \\
\hline $0921+1423$ & A 795 & 0.1357 & 3 & II - Ш & 16.29 & 16.31 & 4.610 \\
\hline $1014+3917$ & A 963 & 0.206 & 3 & II & 16.97 & 17.06 & 4.791 \\
\hline $1058-2227$ & A 1146 & 0.137 & 4 & I - II & 15.98 & 16.23 & 4.614 \\
\hline $1215+2057$ & A 1514 & 0.200 & 3 & II - III & 17.05 & 17.07 & 4.778 \\
\hline $1226+4758$ & A 1550 & 0.2545 & 3 & $\overline{\mathrm{II}}-\overline{\mathrm{III}}$ & 17.34 & 17.36 & 4.883 \\
\hline $1330+5049$ & A 1758 & 0.281 & 3 & ШI & 17.51 & 17.36 & 4.926 \\
\hline $1331+2030$ & A 1759 & 0.168 & 3 & Ш & 17.07 & 16.92 & 4.702 \\
\hline $1441+3123$ & A 1957 & 0.241 & 3 & (I - II): & 17.63 & 17.83 & 4. 859 \\
\hline $1442+3128$ & A 1961 & 0.236 & 3 & II - III & 17.14 & 17.16 & 4.850 \\
\hline $1537+3435$ & A 2111 & 0.228 & 3 & Ш & 17.27 & 17.12 & 4. 835 \\
\hline $1540+6625$ & A 2125 & 0.2465 & 4 & II - III & 17.07 & 17.14 & 4.869 \\
\hline $0939+0912$ & ... & 0.206 & (3) & II - III & 17.15 & 17.17 & 4. 791 \\
\hline
\end{tabular}




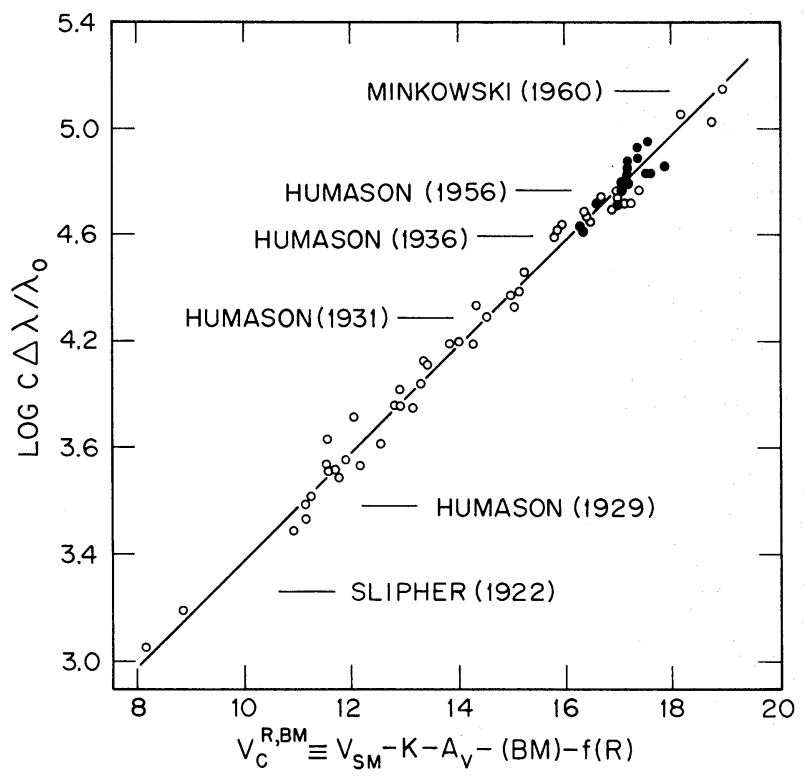

FIG. 4.-The Hubble diagram using fully corrected magnitudes (aperture effect, $K$-effect, absorption, Bautz-Morgan type, and richness) with the same coding as in Fig. 2. The line has the equation $V_{c}^{R, \mathrm{BM}}=5 \log c z-6.865$.

The mean magnitude difference $M_{v}{ }^{R}$, BM between the open and closed circles is now reduced to $0.034 \pm$ $0.076 \mathrm{mag}$, which is negligible. The dispersion in $M_{c}{ }^{R, \mathrm{BM}}$ for the total sample is $0.276 \mathrm{mag}(n=65)$.

\section{b) General Comments}

The new material is too limited to justify a new discussion of the formal value of $q_{0}$. However, from previous solutions, it is clear that $q_{0}$ (formal) $=$ $+1 \pm \sim 0.3$ (with no evolutionary correction applied) will fit the new data well. In particular, there is no indication from the closed circles in Figure 4 that the points are curving from the $q_{0}$ (formal) $=+1$ line toward fainter magnitudes, nor that 3C 295 is abnormally bright. We do not confirm the conclusions of Gunn and Oke (1975) in these two matters for reasons which are not understood at the moment. Much more data are needed at considerably larger redshifts to make progress in these problems, at which time a detailed comparison of the two independent sets of data should be profitable.

The expectations are good that such data will soon be available. The markings in Figure 4 show the progressive extension of the diagram from Slipher's last observations in 1922, through Humason's redshift of NGC 7619 in 1929, to his very large advances in 1931, 1936, and 1956, and finally to Minkowski's (1960) last point at 3C 295. The Humason 1956 limit, which is the cutoff for photographic absorption line spectra, has been the effective limit until very recent years. The three points beyond $\log c z=5$ are all special cases: 3C 295 was measured by Minkowski on the basis of a single very strong $\lambda 3727$ emission line, and the redshifts for $0024+1654$ and $1447+2617$ were obtained photometrically by Baum (1962) and later by Oke (1971). But the limit has now been transcended by new instruments, including, among others, Oke's multichannel scanner, Wampler and Robinson's image-dissector scanner (IDS), and our SIT-prism spectrograph. Recent absorption-line measurements of $z=0.64$ for 3C 123 by Spinrad (1975), using the IDS, and $z=0.53$ for 3 C 330 by us show what can routinely be expected in the next few years, and offer considerable new hope for the resolution of the problem of $q_{0}$ via the Hubble diagram, plus necessary evolutionary corrections.

This work was supported in part by the National Science Foundation under grant GP-36450 X to the Carnegie Institution of Washington.

\section{REFERENCES}

Abell, G. O. 1958, Ap. J. Suppl., 3, 211.

Baum, W. A. 1962, in Problems of Extragalactic Research, ed. G. C. McVittie (New York: Macmillan), p. 390.

Eddington, A. S. 1922, Mathematical Theory of Relativity (Cambridge: Cambridge University Press).

Gunn, J. E., and Oke, J. B. 1975, Ap. J., 195, 255.

Humason, M. L. 1929, Proc. Nat. Acad. Sci., 15, 167.

$$
\text { 1931, Ap. J. 74, } 35 .
$$

1936, ibid., 83, 10.

1957, in Carnegie Yrb., Vol. 56 (Washington: Carnegie Institution of Washington), p. 62.

Humason, M. L., and Sandage, A. 1957, in Carnegie Yrb., Vol. 56 (Washington: Carnegie Institution of Washington), p. 62 (HS).
Matthews, T. A., and Sandage, A. 1963, Ap. J., 138, 30

Minkowski, R. 1960, Ap.J., 132, 908.

Oke, J. B. 1971, Ap. J., 170, 193.

Robertson, H. P. 1955, Pub. A.S.P., 67, 82.

Sandage, A. 1972a, Ap. J., 173, 485 (Paper I).

- 1972b, ibid., 178, 1 (Paper II).

- 1973, ibid., 183, 711 (Paper V)

-. 1975, ibid., 202, 563 (Paper VIII).

Sandage, A., and Hardy, E. 1973, Ap. J., 183, 743.

Slipher, V. M. 1922, in Eddington (1922).

Spinrad, H. 1975, Ap. J. (Letters), 199, L3.

Westphal, J. A., Kristian, J., and Sandage, A. 1975, Ap. J. (Letters), 197, L95 (WKS).

Whitford, A. E. 1971, Ap. J., 169, 215.

Allan Sandage and Jerome Kristian: Hale Observatories, 813 Santa Barbara Street, Pasadena, CA 91101

JAMES A. WestPhal: California Institute of Technology, Geological and Planetary Sciences (170-25), Pasadena, CA 91125 